\title{
Safety of gastric lavage using nasogastric ryle's tube in pesticide poisoning
}

\author{
Uday Bhan Bhardwaj, Anand Subramaniyan, Ashish Bhalla, Navneet Sharma, Surjit Singh* \\ Department of Internal Medicine, Postgraduate Institute of Medical Education and Research, Chandigarh, India; *Corresponding \\ Author: surjit51@hotmail.com, surjit51200@yahoo.co.in
}

Received 13 April 2011; revised 1 June 2011; accepted 10 June 2011.

\begin{abstract}
Objective: Gastric lavage is mandatory irrespective of nature in all patients with acute poisoning in India. Present study was undertaken with aim whether lavage done using naso-gastric Ryle's tube and small aliquots of water or normal saline is safe. Patients and Methods: All the patients above 12 years of age admitted consecutively with pesticide ingestion or exposure between July 2004 to June 2005 were studied with respect to complications associated with lavage using Ryle's tube. Forty five patients were admitted directly to our hospital and lavage was undertaken using Ryle's tube (16F ) with $100-200 \mathrm{~mL}$ of aliquots till $1-1.5$ liters of fluid was lavaged, with prophylactic endotracheal intubation in patients with Glasgow coma scale (GCS) < 10 (group I). The incidence of complications related to lavage in group I was compared to that in $\mathbf{5 3}$ patients admitted during same period with pesticide poisoning but lavaged outside using nasogastric Ryle's tube and referred to our institute (group II). Results: The significant complications observed in group I were significant drop in $\mathrm{SaO}_{2}$ (6 patients) laryngospasm, tachycardia, electrolyte imbalance and tube getting struck in throat (one each). In one patient in group I (had no prophylactic intubation though GCS 3) In group II, 7 had aspiration pneumonia (no prophylactic intubation). Other significant complication was drop in $\mathrm{SaO}_{2}$ during lavage. None of them had any serious life threatening complication. Conclusion: Gastric lavage carried out using naso-gastric Ryle's tube and small aliquots of water or normal saline is relatively safe in patients with pesticide poisoning when combined with prophylactic endotracheal intubation in patients with GCS $<10$. In absence of pro-
\end{abstract}

phylactic intubation, risk of aspiration is there. However aspiration pneumonia is generally mild and not life threatening.

Keywords: Pesticide Poisoning; Gastric Lavage; Nasogastric Ryle's Tube; Complications

\section{INTRODUCTION}

Gastric lavage has to be carried out by treating physician in all patients with acute poisoning in India irrespective of nature, severity of poisoning and time interval between ingestion and arrival to hospital as lavage sample has to be provided to legal authorities in addition to blood, urine and other samples. As per Indian penal code (I.P.C,1973), the attending physician should collect, preserve and seal the evidence related to the case of poisoning such as the gastric lavage fluid, vomiting, faeces, urine etc. for onward transmission to forensic science laboratory for chemical analysis. If doctor deliberately fails to do so he is liable to be punished under section of 201 of I.P.C i.e. causing disappearance of evidence of offence or giving false information to screen offender [1]. This is punishable with imprisonment up to 7 years or fine or both. As per Indian law, attempt to commit suicide is an offence under section 309 of I.P.C which states that whoever attempts to commit suicide and does any act towards the commission of such offence, shall be punished with simple imprisonment for a term which may extend to one year or with fine or with both [2].

This is in contrast to developed countries where suicide is not a criminal offence and lavage is rarely carried out as there is no evidence that it is effective at all [3-7] and can lead to significant morbidity and mortality $[3,8]$. Moreover in developed countries unlike developing countries, drug overdoses are commonest form of poisoning [4]. These carry a low case fatality ratio due to availability of effective antidotes and patients being managed in well equipped hospitals [4-7]. This is unlike developing countries including India, where pesticide 
poisoning is the commonest [9-11]. These cases carry high case fatality ratio as specific antidotes either do not exist or unavailable or unaffordable or only partially effective [11,12]. As gastric lavage in case of poisoning is necessary due to legal reasons in India [1,2] and as studies regarding its safety in pesticide poisoned patients do not exist [13], the present study was undertaken using naso-gastric Ryle's tube with lavage being carried out, using 100 - $200 \mathrm{~mL}$ aliquots of water or normal saline till 1 - 1.5 liters are lavaged, to find its safety. The study was not designed to determine whether gastric lavage removes poison adequately or not.

\section{PATIENTS AND METHODS}

All the patients age 12 or more consecutively admitted to emergency medical services of Nehru Hospital attached to Postgraduate Institute of Medical Education and Research, Chandigarh (India) who had ingested or were accidentally exposed to pesticides and reached our hospital with in 24 hrs were included in the study between July 2004 to June 2005. The patients were divided into 2 groups. Group I included patients with pesticide poisoning who reached our hospital directly and were lavaged by a nurse under supervision of a medical resident or by medical resident. Group II comprised patients who had consumed or exposed to pesticides and had undergone lavage outside our hospital using naso-gastric Ryle's tube and were then referred to us for further management. Only those patients who had symptoms and signs consistent with pesticide poisoning were included in study and all other poisoned patients were excluded.

In group I, gastric lavage was carried out with nasogastric Ryle's tube (16F) and using 100 - $200 \mathrm{~mL}$ aliquots of water or normal saline at a time till $1-1.5$ liters of lavage was completed. Prophylactic endotracheal intubation was carried out in patients with GCS $<10$. In addition to measuring $\mathrm{SaO}_{2}$ by pulse oximeter, arterial blood gases (ABG) were measured before, during and after lavage. In those, where ABG could not be done, $\mathrm{SaO}_{2}$ was continuously determined by pulse oximeter alone. In addition continuous cardiac monitoring was done. A chest x-ray was undertaken before and 4 hours after lavage. Serum electrolytes $(\mathrm{Na}+, \mathrm{K}+, \mathrm{Mg}+$ were measured before and 4 hours after lavage. In group II patients, no lavage was undertaken in our hospital and they were assessed for any complications related to it. It was not possible to know the exact amount of water or normal saline they were lavaged with. However from the hospitals they were referred to us, we checked the lavage practices and it was found that lavage is undertaken generally using smaller aliquots after passing naso-gastric Ryle's tube and fluid volume used generally does not exceed 2 liters.

We could not undertake any tests to find how much compound was removed by lavage from stomach or from blood. The complication rate was determined by determining percentages with confidence interval. The groups were compared using Student t-test. Where ever possible we tried to take patient's consent though it is not necessary as procedure being required by law. The study plan was approved by the ethics committee of the Institute.

\section{RESULTS}

A total of 131 patients with acute poisoning were admitted during this period to our medical emergency. Of these, 98 had history of ingestion or exposure to pesticides and had toxidrome consistent with it. In group I, there were 45 patients. In group II, of 54 one had undergone lavage outside with orogastric tube and was excluded. Thus 98 patients fulfilled the criteria for inclusion in two groups and were taken up for final analysis. Of 45 patients in group I, 40 had ingested with suicidal intent and in 5 poisoning was alleged accidental exposure during spraying (they were found unconscious, ingestion could not be ruled out). Of 53 in group II, 46 had ingested with suicidal intent where as 6 patients were exposed accidentally during spray (as they were found unconscious, ingestion could not be ruled out). In one, circumstances could not be known. In group I, of 11 patients who had GCS $<10$, prophylactic endotracheal intubation was undertaken in 10. In 1 patient with GCS of 3 , medical resident failed to intubate the patient and lavage was undertaken. None in group II had prophylactic endotracheal intubation.

The mean \pm S. $D$ time interval between ingestion or exposure and lavage was $1.95 \pm 2.05$ hours (range 0.5 10 ) in group I and in group II it was $2.5 \pm 3.42$ hours (range 0.5 - 24). The mean \pm S.D age in group I was 28.4 \pm 13.2 (range13 - 65) where is in group II was $27.6 \pm$ 10.8 (range 12 - 68). The compounds ingested or accidentally exposed to in two groups are shown in Table 1.

Table 1. Pesticides ingested or accidentally exposed to in 98 patients.

\begin{tabular}{cccc}
\hline Pesticide consumed & Group I & Group II & Total \\
\hline Organophosphates & 12 & 26 & 38 \\
Aluminum phosphide & 14 & 19 & 33 \\
Carbamates & 4 & 0 & 4 \\
Pyrethroids & 2 & 2 & 4 \\
Unknown & 13 & 6 & 19 \\
Total & 45 & 53 & 98 \\
\hline
\end{tabular}


In group I, mean volume of water or normal saline lavaged was $1108 \mathrm{~mL}$ (range 800 - $1500 \mathrm{~mL}$ ). This could not be known in group II. Hypoxemia i.e. $\mathrm{SaO}_{2}<90 \%$ was present before lavage in 11 (24.4\%) patients where as in group II, 23 (42.6\%) had it at presentation (Table 2). In group $\mathrm{I}$, significant drop in $\mathrm{SaO}_{2}$ (drop in $\mathrm{SaO}_{2}$ to $90 \%$ or lower) during lavage and post lavage was observed in 6 and 2 patients respectively. (Table 3). In 5 patients post lavage $\mathrm{SaO}_{2}$ could not be known as they died before lavage could be completed. All these patients had severe aluminum phosphide poisoning.

The complications which occurred during or following lavage are shown in Table 4. In group I, 44 patients did not develop aspiration pneumonia (prophylactic en- dotracheal intubation carried out in patients with GCS < 10). However, in one patient who had GCS of 3 and prophylactic endotracheal intubation was not undertaken, developed aspiration pneumonia (being defined as appearance of fresh patches of consolidation or increase in patches as seen in post lavage chest x-ray. The other complications observed in this group were sinus tachycardia during lavage in 5 patients (11.1\%), electrolyte imbalance i.e. hyponatremia in one (2.2\%), laryngospasm in one (2.2\%) and in one Ryle's tube got stuck in throat (2.2\%). In group II, 7 (13.2\%) had aspiration pneumonia. None of them had prophylactic endotracheal intubation.

Thirteen patients in group I and 18 in group II died. In

Table 2. Baseline BP, HR and $\mathrm{SaO}_{2}$ at admission in group I (45 patients) and group II (53 patients).

\begin{tabular}{|c|c|c|c|c|c|c|c|c|}
\hline \multirow{2}{*}{ Group } & Systolic $<90^{*}$ & Systolic $>90^{*}$ & $\mathrm{HR}<60^{* *}$ & $\mathrm{HR}>61-100^{* *}$ & $\mathrm{HR}>100^{* *}$ & $\mathrm{SaO}_{2}<90 * * *$ & $\mathrm{SaO}_{2} 90-94 * * *$ & $\mathrm{SaO}_{2}>94 * * *$ \\
\hline & No.\# & No. & No. & No. & No. & No. & No. & No. \\
\hline II & 18 & 35 & 1 & 29 & 23 & 23 & 8 & 22 \\
\hline
\end{tabular}

*Blood Pressure ( mm HG), **Heart rate/min, ***\%O2 saturation, \# Number of patients.

Table 3. $\mathrm{SaO}_{2}$ pre, during lavage and post lavage in group $\mathrm{I}$.

\begin{tabular}{cccc}
\hline $\mathrm{SaO}_{2}$ & $\mathrm{SaO}_{2}$ (admission) & $\mathrm{SaO}_{2}$ (during lavage) & 6 \\
$<90 \%$ & 11 & 3 & 2 \\
$90 \%-94 \%$ & 12 & 36 & 4 \\
$>94 \%$ & 22 & 45 & 34 \\
Total & 45 & 40 \\
\hline
\end{tabular}

Table 4. Complications related to gastric lavage in two groups.

\begin{tabular}{|c|c|c|c|}
\hline Complication & Group I (45) & Group II (53) & Fisher's Exact test p \\
\hline Aspiration pneumonia & $1(2.2 \%)$ & $7(13.2 \%)$ & $0.039 *$ \\
\hline Drop in $\mathrm{SaO}_{2}$ during lavage & $6(13.3 \%)$ & $-* *$ & \\
\hline Laryngospasm & $1(2.2 \%)$ & $-* *$ & \\
\hline Pneumothorax & 0 & 0 & \\
\hline Empyema & 0 & 0 & \\
\hline Tachycardia & $1(2.2 \%)$ & $-* *$ & \\
\hline Ectopics/ST elevation & 0 & $-* *$ & \\
\hline Esophageal perforation & 0 & 0 & \\
\hline Electrolyte imbalance & $1(2.2 \%)$ & $-* *$ & \\
\hline Conjunctival hemorrhage & 0 & 0 & \\
\hline Ryle's tube stuck in throat & $1(2.2 \%)$ & $-* *$ & \\
\hline
\end{tabular}


none, death could be related to lavage associated complications. The important cause of death in both groups was severe aluminum phosphide poisoning.

\section{DISCUSSION}

Gastric lavage has to be carried out in all patients with acute poisoning in India by treating physician irrespective of whether suicidal or accidental as lavage sample has to be provided to legal authorities [1,2]. This is unlike West where suicide is not considered a criminal offence and it is not necessary to collect gastric lavage sample. In West it has fallen out of favor having been found ineffective [2-6]. and can lead to significant morbidity and mortality [1,7] and in recent years, it is much less carried out and if done at all is in either in a consenting or an unconscious intubated patient and is done if poisoning is severe and patient comes very early. However these studies on effectiveness of gastric lavage have been carried out in well equipped Western hospitals in patients with drug overdoses where effective antidotes are available e.g. paracetamol and have low case fatality ratio [4-6]. This is unlike developing countries including India where pesticide poisoning is the commonest [8-10]. These cases carry high mortality e.g. $70 \%$ with aluminum phosphide. The antidotes against pesticides either do not exist or unavailable and unaffordable or partially ineffective. Further, hospitals especially in rural areas are poorly equipped. As the mortality is high and as theoretically gastric lavage may offer some benefit, the practice is common in developing countries $[14,15]$. Further in India, it is necessary to carry it out for medico legal reasons [1,2].

In the present study, we tried to establish the safety of gastric lavage using naso-gastric Ryle's tube with small aliquots of 100 - $200 \mathrm{~mL}$ of water or normal saline being given till 1 - 1.5 liters were lavaged. A significant complication observed was aspiration pneumonia (1/45 in group I and 7/53 in group II) all these patients had not undergone prophylactic endotracheal intubation when patient was obtunded (GCS < 10). The higher incidence of aspiration pneumonia in group II could also have been due to lavage independent factors i.e. deepening of coma with time due to poison itself and transportation of patient with unprotected airway. However in none of these patients it was severe and most of them, unless they had ingested ALP survived (5/8 including one in group I). The other complications in group I were significant drop in oxygen during lavage (6/45) and post lavage (2/40), tachycardia, electrolyte imbalance, laryngospasm during lavage or post lavage was in very small number of patients in group I (one each). We cannot say about these in group II as we could not get any information about these. None of the patients in both groups had serious complications like gastric hemorrhage, gastric perforation, empyema, ST elevation and conjunctival hemorrhage.

These results are unlike those of Eddleston et al. from SriLanka [8] where mainly orogastric tube was predominantly used with large volumes resulting in major complications like severe aspiration, gastric perforation and death. In north-west India where this tertiary centre is located, in majority of peripheral hospitals usually nasogastric Ryles tube is used for gastric lavage using small aliquots and total small volume are being though prophylactic intubation is uncommon due to both lack of equipment and trained manpower. Though doctors and nurses do carry lavage commonly at times it is also carried out by non medical staff (unpublished data). This observation however, cannot be extrapolated to hospitals in other parts of India especially in rural areas where Ryle's tube is not always used and lavage is often being carried out by untrained staff with no intubation being carried out.

The study was not designed to know effectiveness of gastric lavage in removing the pesticide and we cannot comment on this. There is need to undertake studies to find whether gastric lavage effectively removes pesticides or not and if it is not effective why it should be necessary to carry out lavage in all patients with acute poisoning in India irrespective of nature, severity and time interval and this will require Governmental action in bringing about an amendment in IPC.

To conclude gastric lavage carried out with nasogastric tube, using small aliquots of water or normal saline with total lavage fluid less thn $2 \mathrm{~L}$ coupled with careful prophylactic intubation in patients with pesticide poisoning is very safe. However, significant risk of aspiration pneumonia remains if prophylactic intubation is not carried out in patients with < GCS 10. Though aspiration does occur if not intubated prphylactically, generally aspiration pneumonia is not severe and doesn't add to mortality. A significant drop in $\mathrm{SaO}_{2}$ during lavage can also occur.

\section{REFERENCES}

[1] Dikshit, P.C. (2007) General toxicology. Textbook of forensic medicine and toxicology. PEEPEE Publishers and Distributors (P) Ltd., New Delhi.

[2] Indian Penal Code (1999) Universals criminal manual. Universal Law Publishing Company Private Ltd., New Delhi.

[3] American Academy of Clinical Toxicology and European Association of Poison Centres and Clinical Toxicologists (2004) Position statement: Gastric lavage. Journal of Toxicology - Clinical Toxicology, 42, 933-943. doi:10.1081/CLT-200045006

[4] Henry, J.A. and Hoffman, J.R. (1998) Continuing con- 
troversy on gut decontamination. Lancet, 352, 420-421. doi:10.1016/S0140-6736(05)79183-2

[5] Bond, G.R. (2002) The role of activated charcoal and gastric emptying in gastrointestinal decontamination: A state of art review. American Journal of Emergency Medicine, 39, 237-286.

[6] Ardagh, M., Flood, D. and Tait, C. (2001) Limiting the use of gastro-intestinal decontamination does not worsen the outcome from deliberate self poisoning. The New Zealand Medical Journal, 114, 423-425.

[7] Gunnell, D., Ho, D.D. and Murray, V. (2004) Medical management of deliberate drug overdose-a neglected area of suicide prevention. Emergency Medicine Journal, 21, 35-38. doi:10.1136/emj.2003.000935

[8] Eddleston, M., et al. (2007) The hazards of gastric lavage for intentional self poisoning in a resource poor location. Clinical Toxicology, 45, 136-143. doi:10.1080/15563650601006009

[9] Singh, S., Wig, N., Chaudhary, D., Sood, N.K. and Sharma, B.K. (1997) Changing pattern of acute poisoning in adult: Experience of a large Northwest Indian hospital (1970-1989). Journal of Association of Physicians of India, 45, 194-197.
[10] Singh, D., Jit, I. and Tyagi, S. (1999) Changing trends in acute poisoning in Chandigarh zone: A 25 years autopsy experience from a tertiary care hospital in northern India. The American Journal of Forensic Medicine and $\mathrm{Pa}$ thology, 20, 203-210. doi:10.1097/00000433-199906000-00019

[11] Eddleston, M. (2000) Pattern and problems of deliberate self poisoning in the developing world. The Quart Journal, 93, 715-731.

[12] Eddleston, M., Senarathna, I., Mohmad, F., Buckley, N., Juszczak, E., et al. (2003) Deaths due to absence of an affordable antitoxin for plant poisoning. Lancet, 362, 1041-1044. doi:10.1016/S0140-6736(03)14415-7

[13] Eddleston, M., Singh, S. and Buckley, N. (2007) Acute organophosphorus poisoning. Clinical Evidence Handbook. British Medical Journal Publishing Group, London, 413-415.

[14] Bhattarai, M.D. (2000) Gastric lavage is perhaps more important in developing countries. BMJ, 320, 711. doi:10.1136/bmj.320.7236.711

[15] Fernando, R. (1998) Management of acute poisoning. The National Poisons Information Centre in Sri Lanka, Colombo. 